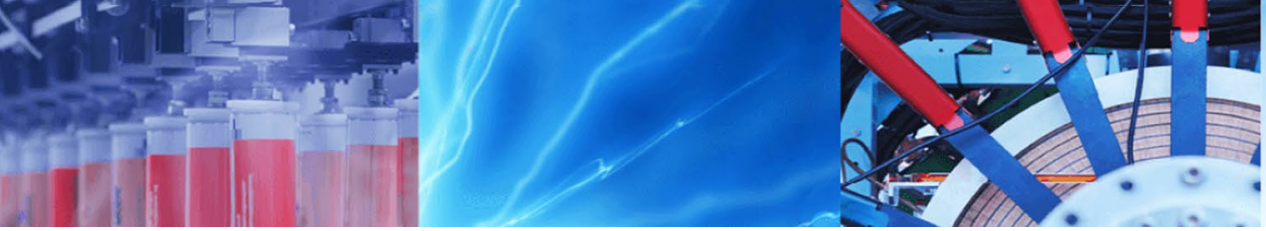

Research Article

\title{
Efficient microwave-assisted extraction of salidroside from Rhodiola crenulata
}

\author{
Rui Liu $^{1,2} \cdot$ Chen Guo ${ }^{2} \cdot$ Mei Feng ${ }^{1} \cdot$ Chun-Zhao Liu' ${ }^{1,2}$
}

Received: 21 October 2020 / Accepted: 22 December 2020 / Published online: 11 January 2021

(C) The Author(s) $2021 \quad$ OPEN

\begin{abstract}
A rapid microwave-assisted extraction (MAE) method was established to extract commercially important salidroside from dry plant materials of Rhodiola crenulata. Several parameters such as extraction time, solid/liquid ratio, extraction temperature and solvent concentration were investigated for efficient salidroside extraction. The estimated maximum salidroside extraction ratio based on parameter optimization using response surface methodology (RSM) reached $2.17 \%$ $(\mathrm{w} / \mathrm{w})$ in $20 \mathrm{~min}$ at optimum conditions of $90^{\circ} \mathrm{C}, 60 \%$ aqueous ethanol and solid to liquid ratio of $2 \mathrm{~g} / 100 \mathrm{~mL}$, which was confirmed to the experimental data. Compared with ultrasonic assistant extraction method (UAE) and heat reflux extraction method (HRE), MAE was the most efficient and effective method in the extraction of salidroside from the dry plant materials. The mechanism on the extraction enhancement using MAE was elucidated further using fourier transform infrared spectroscopy (FTIR) and light microscope.
\end{abstract}

Keywords Fourier transform infrared spectroscopy · Microwave assisted extraction · Response surface method . Rhodiola crenulata Salidroside

\section{Introduction}

Rhodiola crenulata is an important Tibetan plant highly traded as functional food or traditional herb for relieving altitude stress and promoting blood circulation. Recently, many other functions of Rhodiola plants, such as antihypoxia, anticancer and anti-fatigue have been reported [1, 2]. The practical use of Rhodiola root extract includes alleviating mental and physical disorders, as well as reducing the severity of exhaustion after intensive physical exercise. Among the hundreds of active compounds in the extract, salidroside is the main bioactive component in Rhodiola plants [3].

The vast utilities of Rhodiola stimulate the development of many relating biotechniques, including cell culture, tissue culture and extraction technology. The extraction of natural products is essential not only as an evaluation tool for raw materials, but also for the quality control of products. However, traditional methods such as heat reflux extraction (HRE) and Soxhlet extraction are time consuming, energy wasting and less selectivity. New extraction techniques, such as ultrasonic assisted extraction (UAE), microwave assisted extraction (MAE), infrared-assisted extraction and ionic liquids as green extraction solvents, have become important methods for the efficient extraction of salidroside from Rhodiola materials as well as other phytochemicals from plant crops [4-10]. In our previous studies, MAE was also employed in the extraction of solanesol from tobacco leaves [11] and in the extraction of flavonoids from cell cultures of Saussurea medusa Maxim [12]. However, no report on MAE using response

$\triangle$ Mei Feng, qdszxyymnwk@163.com; $\bowtie$ Chun-Zhao Liu, czliu@ipe.ac.cn; czliu@qdu.edu.cn |'State Key Laboratory of Bio-Fibers and Eco-Textiles, Institute of Biochemical Engineering, Qingdao Central Hospital, College of Materials Science and Engineering, Qingdao University, Qingdao 266071, People's Republic of China. ${ }^{2}$ State Key Laboratory of Biochemical Engineering and Key Laboratory of Green Process and Engineering, Institute of Process Engineering, Chinese Academy of Sciences, Beijing 100190, People's Republic of China. 
surface method (RSM) of optimizing salidroside recovery from Rhodiola roots is available.

In present work, the MAE of salidroside from dry root materials of $R$. crenulata was investigated. Preliminary experiments, groups of experiments varying one factor at one time, were performed in order to determine primary extraction conditions for the detailed experimental design. A series of experiments designed by factorial method based on the preliminary conditions was carried out. The results of factorial designed experiments was used to establish a empirical model by response surface method (RSM) to (i) describe the relationships between the studied factors and the response of salidroside extraction ratio, (ii) determine the optimum conditions to achieve the highest extraction ratio. The yield of salidroside as well as extraction efficiency was compared with those of UAE and HRE at above optimum conditions. The mechanism of enhanced extraction process by microwave radiation was investigated by Fourier transform infrared spectroscopy (FTIR) and light microscope analysis of the untreated and treated specimens.

\section{Materials and methods}

\subsection{Materials}

R. crenulata roots collected from Tibetan area were dried at $50^{\circ} \mathrm{C}$ in a hot air oven until constant weight followed by finely pulverized to less than 100-mesh size before the extraction. Salidroside standard ( $>98 \%$ purity) was purchased from Institute for the Control of Pharmaceutical and Biological Products (Beijing, P.R. China). Acetonitrile (HPLC grade) was purchased from Cakedib Laboratoried Ltd. (Georgetown Ont., Canada). Ultrapure water was produced by a Milli-Q system (Millipore, Bedford, MA, USA). Ethanol (analytical reagent grade) was purchased from Beijing Fine Chemical Company.

\subsection{Extraction with different methods}

A household microwave oven was modified for MAE system including water condenser, magnetic stirrer, temperature measurement and time controlling as described previously [11]. With ice water running through the condensation pipe of the MAE system, sample powder was placed into the extraction flask with $100 \mathrm{~mL}$ extraction solvent, and the flask was irradiated automatically by microwave in a pre-setting procedure (a given time of Power On for heating and a given time of Power Off for cooling) in order to keep a desired extraction temperature $(60,80$, $100^{\circ} \mathrm{C}$ ) at a microwave power of $700 \mathrm{~W}$.
UAE was conducted in an ultrasonic bath (KQ2200, Shanghai Cany Precisin Instrument Co., Ltd., China) with a frequency of $40 \mathrm{kHz}$ and a power of $100 \mathrm{~W}$, and the temperature was kept at $60^{\circ} \mathrm{C} .5 \mathrm{~g}$ sample powder was extracted with $100 \mathrm{~mL} 70 \%$ ethanol in a three-neck flask with a reflux condenser for different time.

HRE was performed in $250 \mathrm{~mL}$ flask with a stirring apparatus and a reflux condenser. $5 \mathrm{~g}$ sample powder was added into the flask with $100 \mathrm{~mL} 75 \%$ ethanol. Extraction was carried out with shaking at $60^{\circ} \mathrm{C}$ for different time.

The resulted extract was centrifuged for $5 \mathrm{~min}$ at $10,000 \mathrm{rpm}$ to remove any solid debris. The supernatant was stored at $4{ }^{\circ} \mathrm{C}$ for HPLC analysis.

\subsection{Single-factor experiments of MAE}

For the influence of microwave radiation period on the yield of salidroside with MAE, $5 \mathrm{~g}$ powder sample was extracted with $100 \mathrm{~mL} 75 \%$ ethanol solvent in the MAE system. The microwave radiation period was $0.5,2,4,8$, 12,20 and $30 \mathrm{~min}$, respectively.

For the influence of ethanol concentration on the yield of salidroside with MAE, $5 \mathrm{~g}$ powder sample was extracted with $100 \mathrm{~mL}$ extraction solvent for $20 \mathrm{~min}$ in the MAE system. Various ethanol concentrations $(0 \%, 25 \%, 50 \%, 75 \%$ and $95 \% ; \mathrm{v} / \mathrm{v}$ ) were employed as extraction solvents.

For the influence of solid to liquid ratio on the yield of salidroside with $M A E$, sample powder weighted at 20,10 , 5, 3.5, 2 and $1 \mathrm{~g}$ was extracted with $100 \mathrm{~mL} \mathrm{75 \%} \mathrm{ethanol}$ for $20 \mathrm{~min}$ in the MAE system, respectively.

\subsection{Response surface method (RSM) of MAE}

Based on the above single-factor experiments of MAE, factorial design was carried out to assess the influence of main factors on extraction ratio of salidroside in order to select the optimal working conditions of MAE. The factors included in the factorial design were as follows: ethanol concentration (A), solid to liquid ratio (B), temperature (C) and extraction time (D), which were shown in Table 1 with their levels.

After performing a screening of the factors with the factorial design for the extraction ratio of salidroside, a response surface analysis statistical procedure was employed to achieve the highest extraction ratio. RSM is employed for a model to describe the process behavior of MAE and to optimize the extraction condition. This design is represented by a second-order polynomial regression model (1):

$Y=b_{0}+\sum b_{i} x_{i}+\sum b_{i i} x_{i}^{2}+\sum b_{i j} x_{i} x_{j}+\varepsilon$

The tested factors were coded according to the following regression Eq. (2): 
Table 1 Four factors and corresponding levels

\begin{tabular}{lllll}
\hline Factors & Symbols & \multicolumn{2}{l}{ Actual levels } \\
\cline { 3 - 5 } & & Low $(-)$ & Standard (0) & High $(+)$ \\
\hline Ethanol $(\%, \mathrm{v} / \mathrm{v})$ & $\mathrm{A}$ & 60 & 75 & 90 \\
$\begin{array}{l}\text { Solid/liquid ratio } \\
\quad(\mathrm{g} / 100 \mathrm{~mL})\end{array}$ & $\mathrm{B}$ & 2 & 3.5 & 5 \\
$\begin{array}{l}\text { Temperature }\left({ }^{\circ} \mathrm{C}\right) \\
\text { Extraction time }(\min )\end{array}$ & $\mathrm{C}$ & 70 & 80 & 90 \\
\hline
\end{tabular}

$x_{i}=\frac{X_{i}-X_{0}}{\Delta X_{i}}$

where $x_{i}$ is the dimensionless variable, $X_{i}$ is the actual value of the independent variable, $X_{0}$ is the actual value of the independent variable $i$ at the central point, and $\Delta X_{i}$ is the step change of $X_{i}$ corresponding to a unit variation of the dimensionless value.

\subsection{Analysis}

The untreated and treated samples were dried and kept in a desiccator over silica gel prior to the FTIR analysis by a JASCO FTIR-660 plus spectrometer. The microscope pictures of the samples were taken by an OLYMPUS CX31 light microscope.

The extracts were analyzed by Agilent 1100 HPLC system equipped with an Agilent quaternary pump, an on-line solvent vacuum degasser, an auto sampler and a UV-detector. The data were acquired and processed by means of HP Chemstation software. Individual extract was separated on an RP- $\mathrm{C}_{18}$ column $(250 \mathrm{~mm} \times 4.6 \mathrm{~mm}, 5 \mu \mathrm{m})$ fitted with an Allech $\mathrm{C}_{18}$ pre-column $(8 \mathrm{~mm} \times 4.6 \mathrm{~mm}, 5 \mu \mathrm{m})$. The column temperature was set at $25^{\circ} \mathrm{C}$. The mobile phase was $95 \%$ aqueous acetonitrile at a flow ratio of $1 \mathrm{~mL} / \mathrm{min}$, and the injection volume was $10 \mu \mathrm{L}$. The column eluent was monitored by a UV detector at $276 \mathrm{~nm}$ for salidroside determination. All samples were filtered through $0.22 \mu \mathrm{m}$ filters before use.

The result are expressed as salidroside yield:

$Y(\%)=C_{\text {salidroside }} \times V / m_{\text {Rhodiola }} \times 100 \%$

where $C_{\text {salidroside }}$ is salidroside concentration $(\mathrm{mg} / \mathrm{mL})$ in extract, $V$ is solvent volume $(\mathrm{L})$ and $m_{\text {Rhodiola }}$ is sample weight (mg).

All the experiments were repeated twice, and all values were the means of replicates.

\section{Results and discussion}

\subsection{Single-factor experiments}

As shown in Fig. 1a, the crude extract sample was separated efficiently under the optimized HPLC conditions, and salidroside was quantified concisely for MAE study.

As shown in Fig. 1b. The extraction ratio of salidroside increased with time and reached $2.21 \%$ at $20 \mathrm{~min}$. This may be attributed to the fact that the increasing extraction time caused an increase in reactive sites, resulting in improving extraction yield [13]. Longer extraction time gave little higher extraction yield, but this resulted in time and energy consuming. Therefore, 20 min was selected as the optimal microwave radiation duration for further experiments.

$75 \%(v / v)$ ethanol was confirmed as prefer concentration for further study because of appearance of a peak extraction yield at this concentration as demonstrated in Fig. 1c. An addition of water to ethanol accelerated the mass transfer between the solid and liquid by increasing the permeability of the plant matrix and disrupted the bonding between the plant matrix and solutes [14]. However, the extraction yield declined with increasing ethanol proportion beyond $75 \%(\mathrm{v} / \mathrm{v})$ due that the increased polarity of the extraction solvent with water addition reduced the solubility of phenolic compounds in the aqueous ethanol solution.

As shown in Fig. 1d, the extraction yield decreased sharply with increasing the solid to liquid ratio. The decreased solid to liquid ratio significantly limited diffusivity of the solvent into cells, thus inhibiting the release of the salidroside from the cells. Further decrease of the solid to liquid ratio did not significantly improve the extraction yield, which is congruent with the reports of Chen et al.[13].

\subsection{Factorial design and response surface modeling of MAE}

The statistical methodologies in experimental design of biotechnological processes are increasingly used for the optimization steps. The classical method of "one-variable-at-a-time" bioprocess design is effective in some situations. However, response surface method (RSM) is more effective in the experimental design when there are several factors involved and these factors have combined effect $[15,16]$. Based on the above results of the preliminary experiments, further experiments designed by factorial method were carried out and RSM was applied in the establishment of extraction model. The 

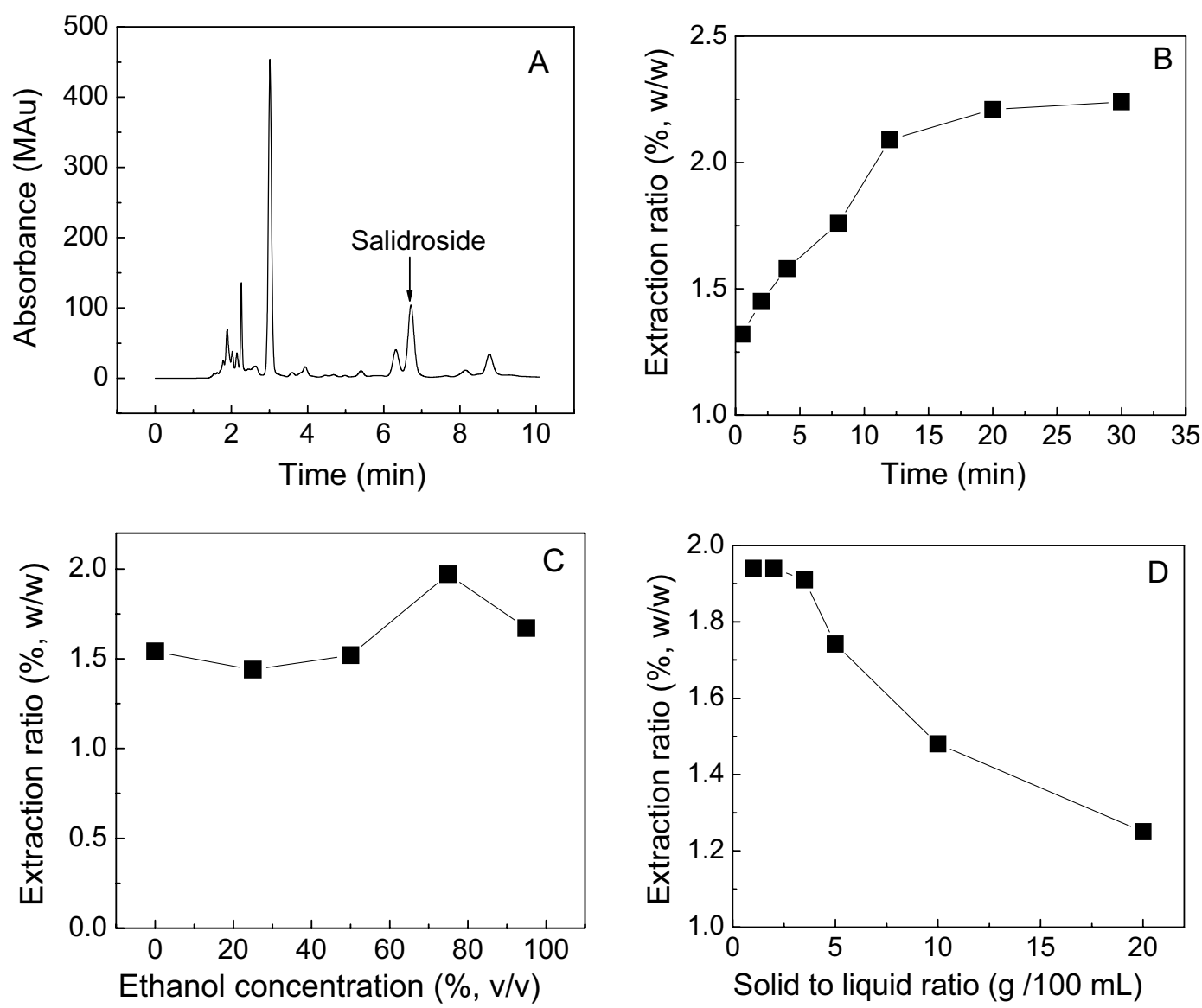

Fig. 1 Separation of salidroside by RP-HPLC chromatography (a) and effect of microwave radiation duration (b), ethanol concentration (c) and solid to liquid ratio (d) on salidroside extraction ratio from $R$. crenulata

experimental design matrix and the recoveries obtained in each run were shown in Table 2.

The analysis of variance (ANOVA) was applied for the determination of significant parameters and for estimation of specific extraction ratio as a function of concentration of ethanol (A), sample/solvent ratio (B), temperature (C) and extraction time (D). The ANOVA reported in Table 3 shows the significant coefficients of independent factors including $\mathrm{B}(p<0.0001), \mathrm{C}(p<0.0005)$ and $\mathrm{D}(p<0.005)$. This indicated that the factors of $B, C$ and $D$ can act as limiting factors. Independent factorial effects are compared in Fig. 2, which displayed average extraction ratio according to the factor level showed in Table 2. Higher differences between levels of one factor indicated more significant influence of this factor on the target.

According to Fig. 2 , it was noticed that the ethanol concentration had the least significant influence shown by the similar extraction ratio with several levels. This correlated with the $p$ values calculated in Table 3. The insignificant influence of ethanol concentration on the process can be explained by its polarity. Generally, polar and polarizable solvents with relative high dielectric constants have the capability of absorption on microwave radiation. Nonpolar and poorly polarizable solvents with low dielectric constants are transparent to microwaves. Therefore, the capability, the extent/depth of penetration and the efficiency of heating by microwaves depends on the polar nature and the dielectric property of the solvent. In this current study, the polar solvent (water and ethanol) were selected as extractant in MAE. The dielectric constant of the mixed solvent was calculated by the following equation:

$\varepsilon_{r}^{1 / 3}=\sum\left(V_{i} / V\right) \times \varepsilon_{r i}^{1 / 3}$

where $\varepsilon_{r}$ is the dielectric constant of mixed solvent, $V_{i}$ is the volume of $i$ solvent, $V$ is the total volume of mixed solvent, $\varepsilon_{r i}$ is the dielectric constant of $i$ solvent. Water $(\varepsilon=81.5)$ and ethanol $(\varepsilon=25.7)$ are good absorber of microwave energy. Also, the dielectric constant of $60 \%, 75 \%$ and $90 \%$ ethanol according to Scheme 5 indicated that all of them are good absorbers of microwave energy. Therefore, the difference between levels of ethanol concentration presents less recognized than that of other factors in Fig. 2. 
Table 2 Experimental design matrix in the three levels factorial design and result

\begin{tabular}{llllll}
\hline No & A & B & C & D & Salidroside (\%) \\
\hline 1 & 90 & 5 & 90 & 20 & 1.94 \\
2 & 90 & 5 & 70 & 20 & 1.60 \\
3 & 75 & 3.5 & 80 & 17.5 & 1.93 \\
4 & 90 & 5 & 70 & 15 & 1.56 \\
5 & 60 & 5 & 70 & 20 & 1.60 \\
6 & 60 & 2 & 70 & 20 & 2.16 \\
7 & 60 & 5 & 70 & 15 & 1.69 \\
8 & 60 & 5 & 90 & 15 & 1.86 \\
9 & 75 & 3.5 & 80 & 17.5 & 1.89 \\
10 & 90 & 2 & 90 & 20 & 2.23 \\
11 & 75 & 3.5 & 80 & 17.5 & 1.86 \\
12 & 90 & 2 & 70 & 20 & 2.19 \\
13 & 90 & 2 & 70 & 15 & 2.10 \\
14 & 60 & 5 & 90 & 20 & 2.09 \\
15 & 60 & 2 & 90 & 20 & 2.24 \\
16 & 60 & 2 & 90 & 15 & 2.01 \\
17 & 60 & 2 & 70 & 15 & 1.92 \\
18 & 90 & 2 & 90 & 15 & 2.13 \\
19 & 90 & 5 & 90 & 15 & 1.80 \\
\hline
\end{tabular}

Table 3 revealed that significant interaction between ethanol concentration and solid/liquid ratio $(p<0.05)$ and significant interaction between solid/liquid ratio and temperature $(p<0.005)$. These interactions are difficult to be obtained by the "one variable at a time" approach. However, the RSM can be used to simulate the effect of these independent variables and their interactions very well. As shown in Fig. 3a, the extraction yield noticeably increased with respect to the increase of extraction temperature. The diffusivity of targeted compounds from solid particles to solvent can be improved by elevated temperature. In addition, surface tension and solvent viscosity also decrease with the increasing temperature which improves sample wetting and matrix penetration. As a result, the solvent had higher capacity to solubilize the active compounds at higher temperature. Because the extraction solvent is evaporated intensively at high temperature, the extraction temperature over $90^{\circ} \mathrm{C}$ was not discussed in the current study. As shown in Fig. 3b, the lower solid/liquid ratio favored a higher extraction yield. The extraction yield was enhanced at a lower solid/liquid ratio because of increasing diffusivity of the solvent into cells to release more targeted compounds into the extraction solvent.

The equation obtained for the RSM model was:

$$
\begin{aligned}
Y= & 1.84619-0.30397^{*} b-0.0051^{*} c+0.024467^{*} d \\
& -0.00188^{*} a * b+0.004087^{*} b^{*} c
\end{aligned}
$$

where $Y$ is the extraction ratio of salidrosade, $a, b, c$ and $d$ are calculated by Eq. (2) from A, B, C and D which are the coded settings in Table 1 . The computed $p$-value $(<0.0001)$ and $R$-squared value $\left(R^{2}=0.9413\right)$ of this model indicate that this model is highly significant at high confidence level (>99\%). The ANOVA suggests that the model equation is adequate to predict the MAE process of salidroside. The results of regression analysis predicted that the highest extraction ratio of salidroside $(2.17 \%)$ was obtained at the following extraction conditions: ethanol concentration: $60 \%$; solid /liquid ratio: $2 \mathrm{~g} / 100 \mathrm{ml}$; extraction temperature: $90^{\circ} \mathrm{C}$; and extraction time: $20 \mathrm{~min}$. The predicted result was close to the experimental result (2.24\%). Response surface modeling for the operating condition optimization was also demonstrated
Table 3 ANOVA for selected factorial model

\begin{tabular}{lllllll}
\hline Source & SS & DF & MS & F-value & $p$-value & \\
\hline Model & 0.7859 & 6 & 0.1310 & 29.3769 & $<0.0001$ & Significant \\
A-ethanol & 0.0000 & 1 & 0.0000 & 0.0001 & 0.9909 & Not significant \\
$\begin{array}{l}\text { B-sample mass to } \\
\quad 0.5018\end{array}$ & 1 & 0.5018 & 112.5520 & $<0.0001$ & Significant \\
C-tement ratio & & & & & & \\
D-extraction time & 0.0599 & 1 & 0.0599 & 13.4260 & 0.0037 & Significant \\
AB & 0.0286 & 1 & 0.0286 & 6.4250 & 0.0277 & Significant \\
BC & 0.0601 & 1 & 0.0601 & 13.4857 & 0.0037 & Significant \\
Curvature & 0.0073 & 1 & 0.0073 & 1.6407 & 0.2266 & Not significant \\
Residual & 0.0490 & 11 & 0.0045 & & & \\
Lack of fit & 0.0470 & 9 & 0.0052 & 5.0104 & 0.1774 & not significant \\
Pure error & 0.0021 & 2 & 0.0010 & & & \\
Cor total & 0.8423 & 18 & & & & \\
\hline
\end{tabular}

$R^{2}=0.9413 ;$ Adj $R^{2}=0.9092 ;$ Pred $R^{2}=0.8166 ;$ Adeq Precision $=17.198$

SS sum of squares, $D F$ degrees of freedom, MS mean square 
Fig. 2 Independent factorial effect of ethanol concentration (A), sample/solvent ratio (B), temperature $(C)$ and the extraction time (D) on salidroside extraction ratio in factorial design
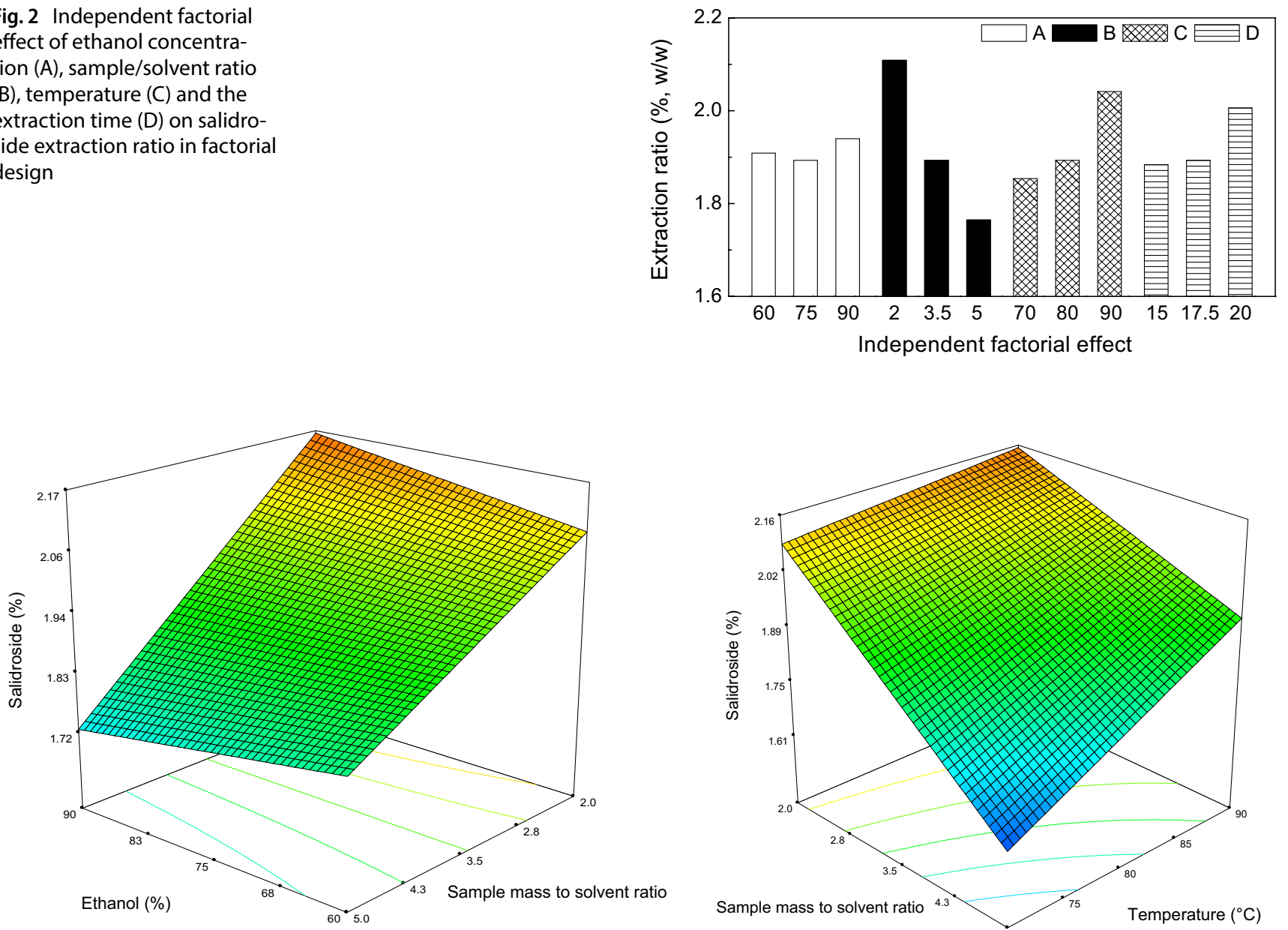

A

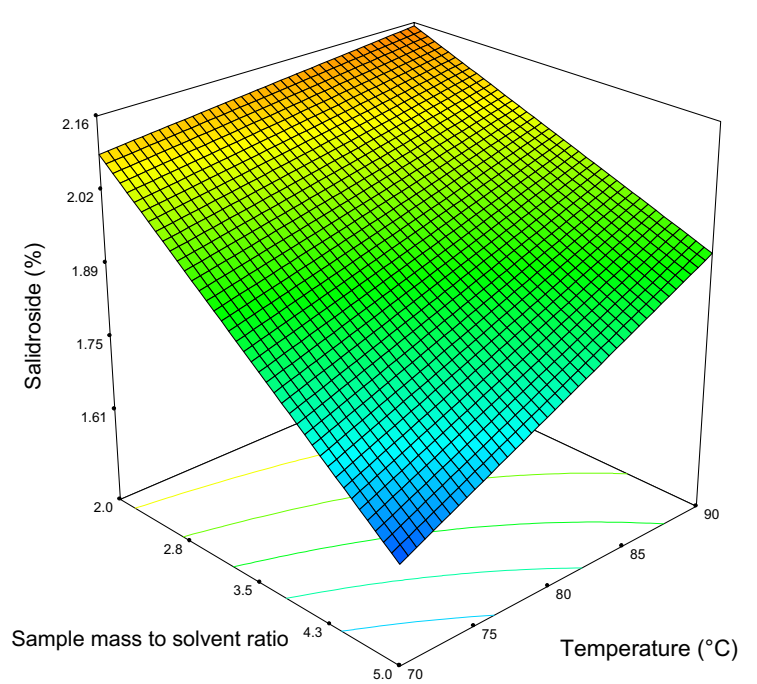

B

Fig. 3 Response surface estimated from factorial design by plotting proportion of ethanol vs. sample mass to solvent ratio (A), as well as sample mass to solvent ratio vs. temperature (B)

in improving polyphenols extraction process from apple skins [17].

\subsection{Comparison of MAE with both UAE and HRE}

As shown in Fig. 5, the salidroside extraction ratio reached $2.21 \%$ in 20 min by MAE. While for HRE, it took more than one hours to reach a maximum extraction ratio of $1.764 \%$. Compared with HRE, UAE needed a shorter time $(30 \mathrm{~min})$ to reach a relatively high salidroside extraction ratio of $0.919 \%$, while it is still lower than that by MAE. As a result, the extraction efficiency of MAE is much higher than that of UAE and HRE (Fig. 4). These results indicate that the MAE is a proficient method with a high extraction ratio and high extraction efficiency in the extraction of salidroside from $R$. crenulata. Similar results have reported on extracting chlorogenic acid from flower buds of Lonicera japonica
Thunb [18] and flavonoids from cultured cells of $S$. medusa Maxim [19].

The advantages of MAE compared with other extraction methods are to destruct plant cell wall under microwave radiation. This destruction decreased the diffusion resistance and was favorable to the mass transfer of active components through cell walls [9]. In order to understand the MAE function very well, all samples were examined by FTIR and light microscope, respectively. FTIR is frequently used for investigating the structure of constituents and chemical changes in biomass. As shown in Fig. 5, both lignin characteristic peak (1240 $\mathrm{cm}^{-1}, \mathrm{C}-\mathrm{O}$ of guaiacyl ring) and cellulose characteristic peak $\left(1033 \mathrm{~cm}^{-1}, \mathrm{C}-\mathrm{O}\right.$ stretch) in the treated samples were lower than those of untreated samples. This indicated that the degradability of both lignin and cellulose was happened under the microwave radiation. 

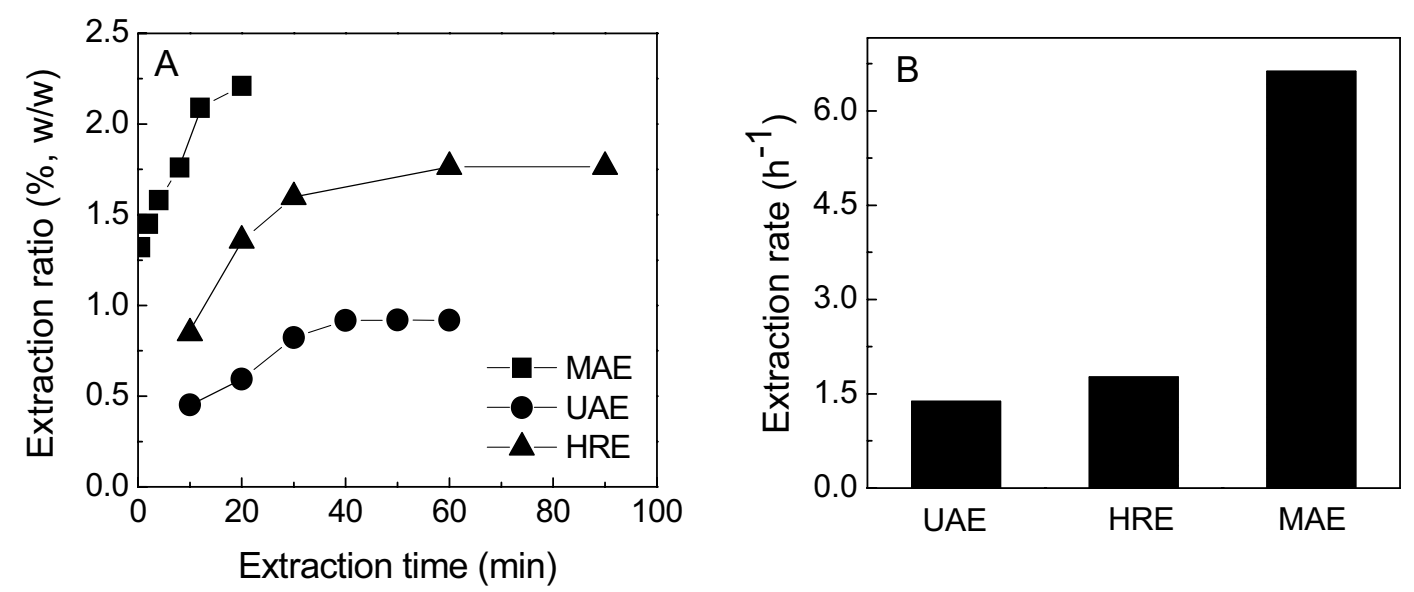

Fig. 4 Effect of different extraction method on salidroside extraction ratio (a) and extraction rate (b) from R. crenulata

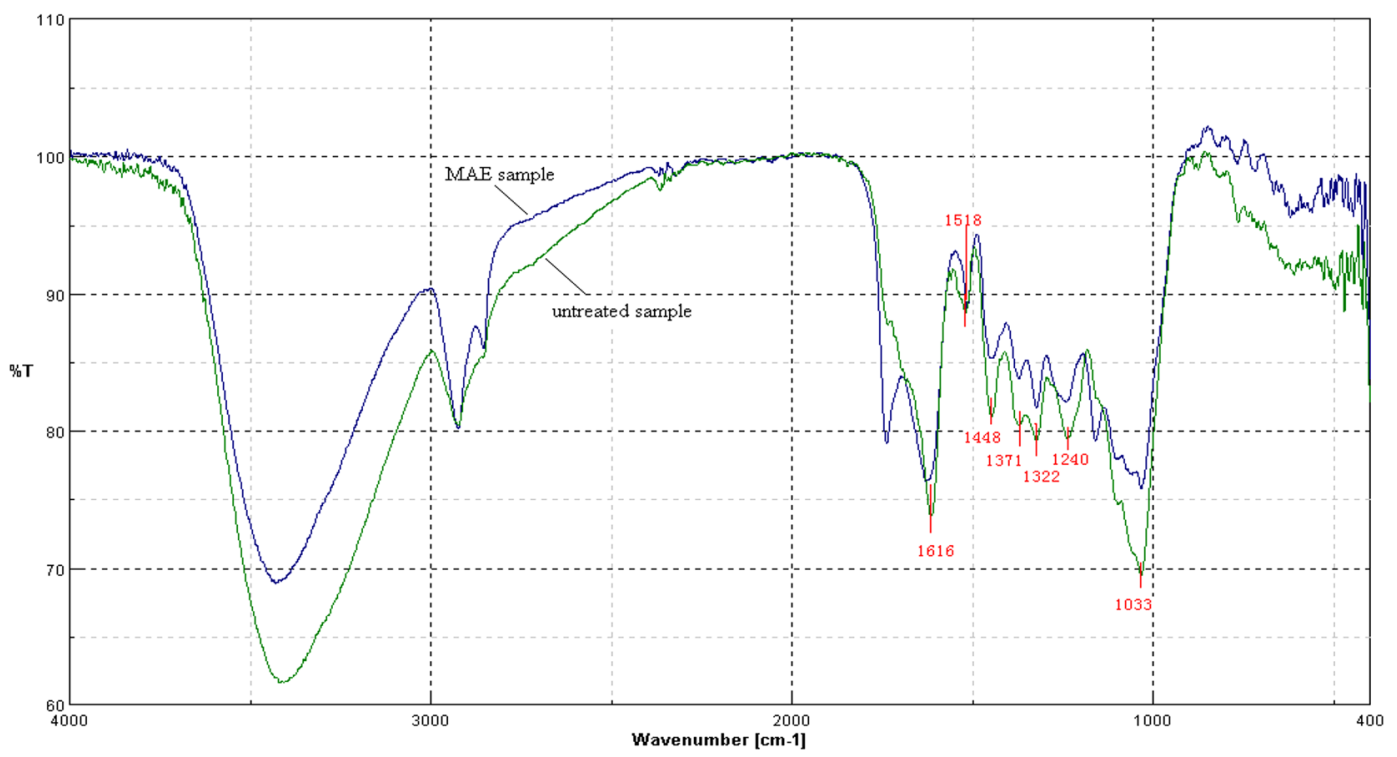

Fig. 5 FTIR spectra of treated and untreated samples of $R$. crenulata

As shown in Fig. 6, the intact cells of the untreated samples exhibited rigid and highly ordered cell walls, and the cell walls exposed to the microwave radiation were ruptured. As a result, the enhanced salidroside extraction efficiency was achieved through the improved mass transfer of the cell constituents through the cell walls.

\section{Conclusions}

RSM was developed successfully to predict the salidroside extraction from $R$. crenulata roots, and the maximum salidroside extraction ratio predicated by RSM under the optimal MAE conditions was consistent to the experimental ones. Compared with UAE and HRE, MAE was the best extraction method with the highest extraction yield and the shortest extraction time. The advantages of MAE were ascribed to the destruction of plant cell walls under microwave radiation which was favorable to the mass transfer of active components through the cell walls through the observation results by FTIR and light microscope. 

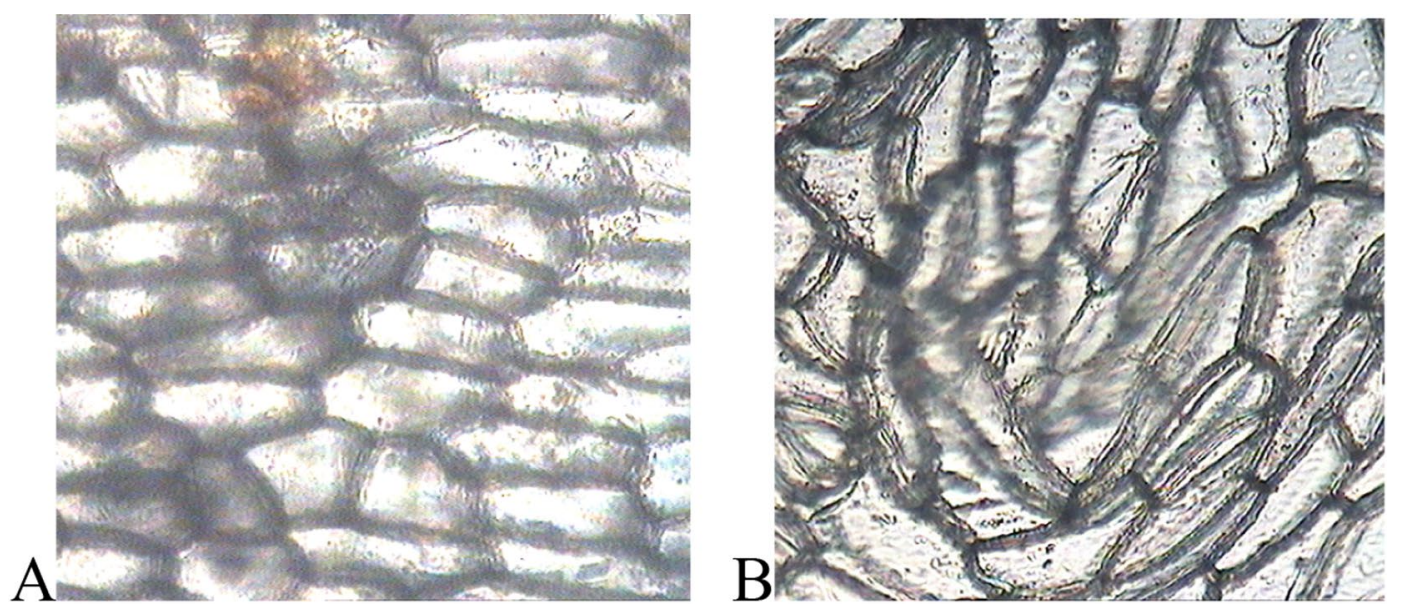

Fig. 6 Light microscope photographs of untreated (a) and treated (b) samples of R. crenulata

Acknowledgements This work was financially supported by the Key Research and Development Project of Shandong Province (2019GSF109079), State Key Laboratory of Bio-Fibers and Eco-Textiles (Qingdao University), and Qingdao Key Health Discipline Development Fund.

\section{Compliance with Ethical Standards}

Conflict of interest All the authors associated with this manuscript declare that there is no conflict of interest.

Open Access This article is licensed under a Creative Commons Attribution 4.0 International License, which permits use, sharing, adaptation, distribution and reproduction in any medium or format, as long as you give appropriate credit to the original author(s) and the source, provide a link to the Creative Commons licence, and indicate if changes were made. The images or other third party material in this article are included in the article's Creative Commons licence, unless indicated otherwise in a credit line to the material. If material is not included in the article's Creative Commons licence and your intended use is not permitted by statutory regulation or exceeds the permitted use, you will need to obtain permission directly from the copyright holder. To view a copy of this licence, visit http://creativecommons .org/licenses/by/4.0/.

\section{References}

1. Majewska A, Grażyna H, Mirosława F, Natalia U, Agnieszka P, Alicja Z, Kuraś M (2006) Antiproliferative and antimitotic effect, S phase accumulation and induction of apoptosis and necrosis after treatment of extract from Rhodiola rosea rhizomes on HL-60 cells. J Ethnopharmacol 103:43-52

2. Shevtsova VA, Zholusb Bl, Shervarlyb VI,Vol'skijbVB, KorovinbYP, Khristichb MP, Roslyakovac NA, Wikman G (2003) A randomized trial of two different doses of a SHR-5 Rhodiola rosea extract versus placebo and control of capacity for mental work. Phytomed 10:95-105

3. Hong HD, Kim JC, Lim TG, Song YR, Cho CW, Jang M (2018) Mixing ratio optimization for functional complex extracts of Rhodiola crenulata,Panax quinquefolius and Astragalus membranaceus using mixture design and verification of immune functional efficacy in animal models. J Funct Foods 40:447-454
4. Hemwimol S, Pavasant P, Shotipruk A (2006) Ultrasound-assisted extraction of anthraquinones from roots of Morinda citrifolia. Ultrason Sonochem 13:543-548

5. Mao Y, Li Y, Yao N (2007) Simultaneous determination of salidroside and tyrosol in extracts of Rhodiola L. by microwave assisted extraction and high-performance liquid chromatography. J Pharm Biomed Anal 45:510-515

6. Đurovića S, Nikolića B, Lukovićb N, Jovanovićb J, Stefanovićb A, Šekuljicac N, Mijind D, Knežević-Jugovićb Z (2018) The impact of high-power ultrasound and microwave on the phenolic acid profile and antioxidant activity of the extract from yellow soybean seeds. Ind Crops Prod 122:223-231

7. Zhang YF, Zhou ZL, Zou L, Chi R (2019) Imidazolium-based ionic liquids with inorganic anions in the extraction of salidroside and tyrosol from Rhodiola: the role of cations and anions on the extraction mechanism. J Mol Liquids 275:136-145

8. Li FJ, Yuan Y, Zhan ZL, Kang LP, Li M, Yang B, Huang LQ (2015) Infrared-assisted extraction of salidroside from the root of Rhodiola crenulata with a novel ionic liquid that dissolves cellulose. RSC Adv 59:47326

9. Hu B, Zhou K, Liu YT, Liu AP, Zhan Q, Han GQ, Liu SL, Yang Y, Zhu YD, Zhu DF (2018) Optimization of microwave-assisted extraction of oil from tiger nut (Cyperus esculentus L.) and its quality evaluation. Ind Crops Prod 115:290-297

10. Gomez AV, Tadinia CC, Biswasc A, Buttrum M, Kim S, Boddu VM, Cheng HN (2019) Microwave-assisted extraction of soluble sugars from banana puree with natural deep eutectic solvents (NADES). LWT: Food Sci Technol 107:79-88

11. Zhou HY, Liu CZ (2006) Microwave-assisted extraction of solanesol from tobacco leaves. J Chromatogr A 1129:135-139

12. Gao M, Song BZ, Liu CZ (2006) Dynamic microwave-assisted extraction of flavonoids from Saussurea medusa Maxim cultured cells. Biochem Eng J 32:79-83

13. Chen C, Zhang B, Huang Q, Fu X, Liu RH (2017) Microwave-assisted extraction of polysaccharides from Moringa oleifera Lam. leaves: characterization and hypoglycemic activity. Ind Crops Prod 100:1-11

14. Muniz-Marquez DZ, Martinez-Avila GC, Wong-Paz JE, BelmaresCerda R, Rodriguez-Herrera R, Aguilar CN (2013) Ultrasoundassisted extraction of phenolic compounds from Laurus nobilis $\mathrm{L}$. and their antioxidant activity. Ultrason Sonochem 20:1149-1154

15. Wang YG, Li YL, Ma XQ, Ren HW, Fan WG, Leng FF, Yang MG, Wang $X L$ (2018) Extraction, purification, and bioactivities analyses 
of polysaccharides from Glycyrrhiza uralensis. Ind Crops Prod 122:596-608

16. Dai LM, Zhang J, Cheng F (2019) Succeeded starch nanocrystals preparation combining heat-moisture treatment with acid hydrolysis. Food Chem 278:350-356

17. Casazza AA, Pettinato M, Perego P (2020) Polyphenols from apple skins: a study on microwave-assisted extraction optimization and exhausted solid characterization. Sep Purif Technol 240:116640
18. Zhang B, Yang RY, Liu CZ (2008) Microwave-assisted extraction of chlorogenic acid from flower buds of Lonicera japonica Thunb. Sep Purif Technol 62:480-483

19. Gao M, Liu CZ (2005) Comparison of techniques for the extraction of flavonoids from cultured cells of Saussurea medusa Maxim. World J Microbiol Biotechnol 21:1461-1463

Publisher's Note Springer Nature remains neutral with regard to jurisdictional claims in published maps and institutional affiliations. 